\title{
Physio-Hematological Response of Healthy Dogs During and after Ovariohysterectomy
}

\author{
Dionesio A. Estopa
}

\begin{abstract}
This study investigated six apparently healthy female dogs as experimental animals for the cardiovascular response and the changes in the hematological parameters subjected to ovariohysterectomy. The animals were examined prior to surgery through heart rate evaluation and complete blood count examination. Heart rate evaluation and Complete Blood Count (CBC) examination were performed before, during and after ovariohysterectomy. A non-parametric test (averages and percentages) were used to compare the variation of heart rate and blood parameters. Results of the study showed that there was a slight reduction of the heart rate during the surgical operation and a significant numerical difference in the values were observed in the blood parameters (RBC, WBC, PCV, MCV, Platelets, Lympocytes and Segmented neutrophils) throughout the experimental period. Red Blood Cell (RBC) count was affected during the intra-operative period slightly below the normal range. Packed Cell Volume (PCV) was also reduced during surgery, but the reduction is still within the normal range. Reduction in the Mean Corpuscular Volume (MCV) was also observed, however, not exceeding beyond the normal range. White Blood Cell (WBC) count however, was observed to be elevated during the surgery but the values of segmented neutrophils were within the normal range. The results of the study revealed that ovariohysterectomy really trigger intra-operative stress. Therefore, a careful evaluation of the patient prior to the surgical operation should be given utmost consideration. Surgical patients with abnormal blood parameters should be stabilized first before proceeding with the said surgical operation. During the intra-operative period, it is highly recommended that the patient should be under intravenous fluid infusion to maintain its homeostasis and acid-base balance.
\end{abstract}

Keywords: Physio-Hematological Response, Ovariohystectomy, Healthy Dogs

\begin{abstract}
Introduction
For so many years, dogs have lived and worked with humans in so many ways that they have earned a unique name "a man's best friend". They also have significant benefits to humans, for instance, acting as a guard, children's playmate or walking companion. Majority of contemporary people with dogs describe their pets as part of the family. Considering the benefits they give, dogs, just like humans, need proper care to prevent them from getting sick or contacting diseases. Diseases or infections can occur regardless of their sex. Ovariohysterectomy or spay, is the most common surgery performed in female dogs; it is the complete surgical removal of the female reproductive tract; the ovaries, oviducts, uterine horns and the uterus is removed. Not only does this procedure prevent dogs or other animals from becoming pregnant, it also helps eliminate the yearly heat cycle.
\end{abstract}

Ovariohysterectomy is one of the techniques used to control sexual behavior and conception aiming at the prevention of uterine diseases, recurrence of vaginal hyperplasia and it is also indicated for animals with diabetes or epilepsy to prevent hormonal influences on therapy.
Surgery is a fascinating branch of therapeutic science which deals with the treatment of disease and injury with operative and manipulative method. But unfortunately, surgery also causes stress, a physiologic response to trauma which can be assessed by blood cell count and heart rate measurement. The intra-operative surgical stress response in dogs has not previously been described. The aim of this study is to investigate the intraoperative surgical stress response and to get an idea of the overall health status of the animals during and after ovariohysterectomy.

\section{Objective of the Study: \\ To compare and evaluate the cardiovascular response during and after ovariohysterectomy and to determine any hematological changes of dogs during and after ovariohysterectomy.}

\begin{abstract}
Materials and Methods
Experimental Animal:

The study population consist of six (6) female privately owned dogs admitted to UEP Veterinary Teaching Hospital for ovariohysterectomy. All dogs were examined to determine if they are fit for surgical operation thru owner interview and clinical examination.
\end{abstract}




\section{Heart Rate Evaluation:}

The heart rate was checked during and after surgery. A comparison and evaluation of the heart rate during and after the surgery were done using stethoscope.

\section{Administration of Anesthetics:}

Atropine sulfate was used as pre-anesthetic agent at $0.04 \mathrm{mg} / \mathrm{kg}$ body weight subcutaneously. After 10 minutes, dogs were given with Zolitel 50 at 7.5 to 10 $\mathrm{mg} / \mathrm{kg}$ dosage for induction of anesthesia. Surgical level of anesthesia was maintained with inhalation of isoflurane mixed in air at 2-3\%. oxygen and air mixture at approximately $20-40 \mathrm{ml} / \mathrm{kg} /$ in a circle breathing system gas anesthesia machine.

\section{Ovariohysterctomy Procedure:}

The dogs were placed in dorsal recumbency and the surgical site was clipped and scrubbed with povidone iodine. Sterile drapes were placed with towel clamps. A ventral midline incision of the skin and subcutaneous layer was made approximately an inch away from the umbilicus and $3-5 \mathrm{~cm}$ in length. The subcutaneous fat was removed to have a clearer view of linea alba. A lift and stab method was used in the linea alba to enter the abdominal cavity. The uterus was located by use of the spay hook. The proper ligament was clamped. Tearing or breaking the suspensory ligament to allow better visualization of the ovary. The ovarian pedicle was clamped and ligated, two ligatures were made. The ovarian pedicle was checked for any bleeding before it was released back to the abdominal cavity. The procedure was repeated on the contra-lateral side. The body wall was closed using simple continuous suture pattern and the skin was closed using interrupted suture pattern. While the patients were under this surgical procedure, the heart rate were checked every fifteen minutes and blood samples were collected for Complete Blood Count (CBC) determination.

\section{Interpretation of the results:}

To measure the variation of heart rate and blood parameters during and after ovariohysterectomy, the data was tabulated and measured with non-parametric statistics using averages and percentages, and was presented in a form of graph.

\section{Results and Discussion}

Heart rate is part of physical examination in getting the vital signs of the surgical patient. Nonetheless, rates outside the normal range, both high and low, are associated with reduced cardiac output. The average heart rates per minute of the six (6) experimental animals before, during and after ovariohysterectomy are given in Table 1. As shown in the same table, the animals are healthy before being subjected to surgery with an average of 103 beats per minute. The heart rate did not change significantly, only a slight reduction of heart rate ( 98 beats per minute) during the surgical operation was observed; a low heart rate is caused by stimulation during surgery and it could be attributed to the surgical level of anesthesia during its intraoperative phase. And on the post-operative evaluation of the heart rate which was conducted 24 hours after the surgical operation, the heart rate was restored to a normal level of 105 beats per minute.

Table 1. The average heart rate per minute of six (6) experimental animals

\begin{tabular}{|l|c|}
\hline BEFORE SURGERY & 103 beats $/ \mathrm{min}$. \\
\hline DURING SURGERY & 98 beats $/ \mathrm{min}$. \\
\hline AFTER SURGERY & 105 beats $/ \mathrm{min}$. \\
\hline NORMAL RANGE & $100-130$ beats $/ \mathrm{min}$. \\
\hline
\end{tabular}

Table 2. Average in the Complete Blood Count $(\mathrm{CBC})$ results of the six (6) experimental animals (normal range is also presented).

\begin{tabular}{|l|l|l|l|l|}
\hline \multirow{2}{*}{ BLOOD COMPONENTS } & \multicolumn{3}{|c|}{ SURGERY } & \multirow{2}{*}{ NORMAL RANGE } \\
\cline { 2 - 4 } & \multicolumn{1}{|c|}{ BEFORE } & DURING & AFTER & \\
\hline $\mathrm{RBC}\left(\times 10^{6} / \mu l\right)$ & 7.5 & 5.8 & 6.5 & $6-9$ \\
\hline $\mathrm{PCV}(\%)$ & 41 & 37 & 39 & $37-55$ \\
\hline $\mathrm{Hgb}(\mathrm{g} / \mathrm{dl})$ & 13.7 & 12.3 & 13 & $12-18$ \\
\hline $\mathrm{MCV}(\mathrm{fl})$ & 65 & 55 & 63 & $60-77$ \\
\hline $\mathrm{MCH}(\mathrm{pg})$ & 18.2 & 21.6 & 20.7 & $13-25$ \\
\hline $\mathrm{MCHC}(\mathrm{g} / \mathrm{dl})$ & 33.2 & 33.2 & 33.2 & $31-36$ \\
\hline
\end{tabular}




\begin{tabular}{|l|l|l|l|l|}
\hline Platelets $\left(\times 10^{5} / \mu l\right)$ & 4.8 & 6.5 & 5.6 & $2-9$ \\
\hline WBC $\left(\times 10^{3} / \mu l\right)$ & 9.8 & 16.5 & 14.6 & $6-15$ \\
\hline Eosinophils $(\%)$ & 6 & 7 & 6 & $2-10$ \\
\hline Basophils $(\%)$ & 0.3 & 0.4 & 0.3 & $0-.5$ \\
\hline Lymphocytes $(\%)$ & 16 & 11 & 13 & $12-30$ \\
\hline Monocytes (\%) & 2 & 1 & 1 & $3-9$ \\
\hline Neutrophils (segmented) & 64 & 70 & 68 & $60-75$ \\
\hline Neutrophils (bands) & 4 & 4 & 5 & $0-4$ \\
\hline
\end{tabular}

RBC Count $\left(\times 10^{6} / \mu l\right)$ :

The RBC count is one parameter in the detection of anemia and is calculated using this formula; $\mathrm{RBC}=\frac{\# \text { of cells counted (average of the two sides })}{(\text { dilution ratio }) \times(\text { volume of one square }) \times(\text { no.of squares counted })}$.

Table 2 shows the average results of the red blood cell (RBC) count before, during and after ovariohysterectomy. Before subjecting the animals to surgery, the red blood cell count was normal (7.5) but it falls below the normal level (5.8) during surgical operation. And on post-operative examination, the red blood cell count was restored to normal level (6.5).

\section{RBC COUNT}

\section{BEFORE EDURING AFTER}

10

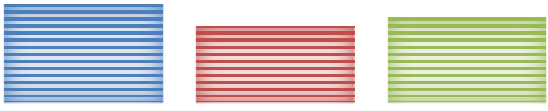

Figure 10.A graphical presentation of RBC Count before, during and after ovariohysterectomy.

The reduction of RBC count was obviously due to inevitable bleeding or loss of blood during the surgical procedure.

Packed Cell Volume (PCV \%):

In Complete Blood Count (CBC), we determine the numbers of $\mathrm{RBC}$ in several different ways and the quickest and easiest is the Packed Cell Volume (PCV). As shown in Table 2, the average packed cell volume of the six (6) experimental animals before the surgery was normal (41\%). During surgical operation, blood examination revealed a slight reduction of Packed Cell Volume (37\%), however, it does not indicate unhealthy condition since it was still within the normal range. On post-operative examination of blood which was conducted 24 hours after the surgery, only a slight increase from $37 \%$ to $39 \%$ was seen.

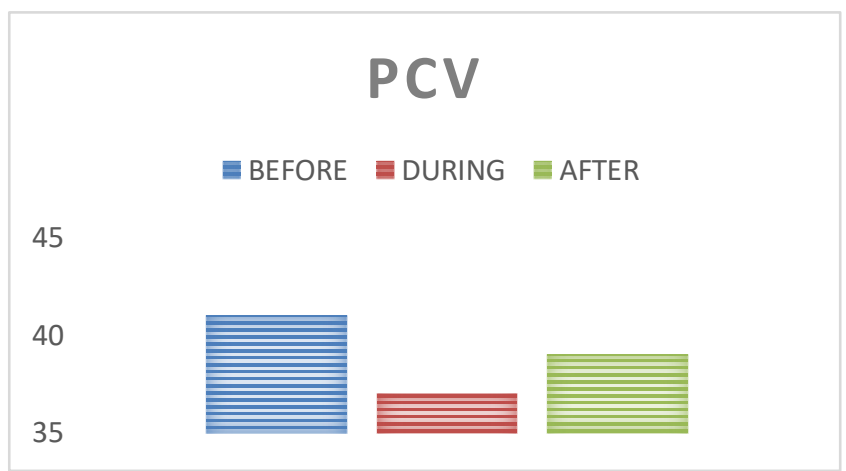

Figure 11.A graphical presentation of PCV before, during and after ovariohysterectomy.

In relation to Red Blood Cells (RBC) and for the fact that we cannot avoid bleeding during surgery, it is thought that Packed Cell Volume (PCV) will also decrease during surgery. But the results of the Packed Cell Volume (PCV) before, during and after remained within the normal range.

\section{HEMOGLOBIN(g/dl):}

The final way we can evaluate the red blood cells (RBC) is by quantifying the hemoglobin present. The average hemoglobin level before, during and after ovariohysterectomy was presented in Table 2 where it was shown that the dogs' hemoglobin level before surgery was 13.7 which was normal. As shown in the same table, decreased hemoglobin level (12.3) was seen during the surgical operation, however, the value was still within the normal range (12-18) but if the cells contain less than the normal amount of hemoglobin, it could mean a sign of anemia. But then on the following day after the surgery, blood examination revealed a slight increase of hemoglobin level (13). 


\section{HEMOGLOBIN}

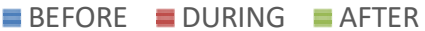

15

10
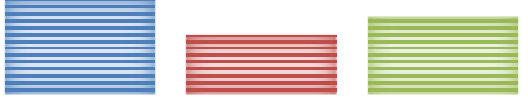

Figure 12.A graphical presentation of Hemoglobin before, during and after ovariohysterectomy.

MCV (fl):

Mean corpuscular volume (MCV) is the average actual size of the red blood cells in circulation and is measured in femtoliters (fl). It was calculated by multiplying the PCV value by 10 and dividing by the red blood cell count

$\left(\mathrm{MCV}=\frac{P C V \times 10}{R B C\left(10^{6}\right)}\right)$.

Normal mean corpuscular volume (MCV) was seen before ovariohysterectomy with an average of $65 \mathrm{fl}$. $\mathrm{MCV}$, as well $\mathrm{MCH}$ and $\mathrm{MCHC}$, reflect to the average values of red blood cell (RBC) count. That is why based on the result of the study, the MCV also falls below the normal range during surgery, $55 \mathrm{fl}$.But then, on the post-surgical blood examination the MCV values returned to normal, $63 \mathrm{fl}$.

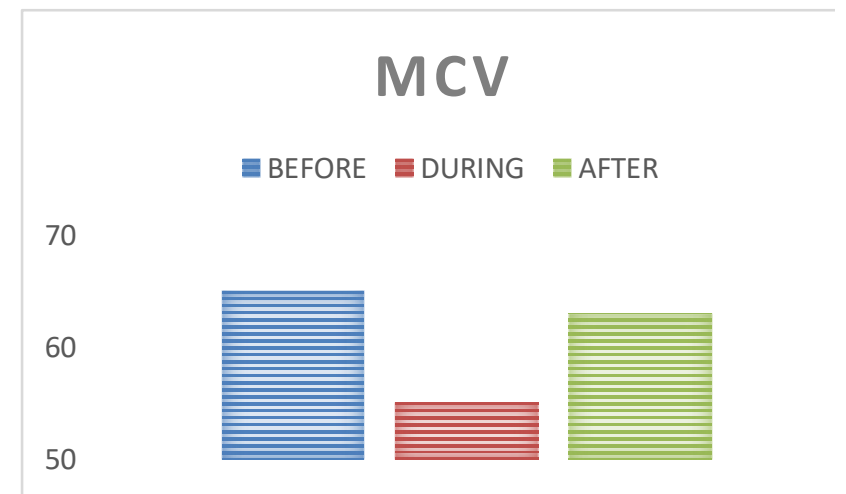

Figure 13.A graphical presentation of MCV before, during and after ovariohysterectomy.

MCH (pg):

Mean corpuscular hemoglobin $(\mathrm{MCH})$ is a measure of the average weight of hemoglobin found in the circulating red blood cells and is measured in picograms (pg). It was calculated by multiplying the hemoglobin concentration by 10 and then dividing by the total red blood cell count $M C H=\frac{H g b \times 10}{R B C\left(10^{6}\right)}$.

A normal mean corpuscular hemoglobin $(\mathrm{MCH})$ were seen before, during and after ovariohysterectomy. It was presented in table 2that in the preoperative values of $\mathrm{MCH}$ was 18.2. A slight increase in the
$\mathrm{MCH}$ valuesduring the surgical operation was seen and on the post-surgical examination, the $\mathrm{MCH}$ slightly decreased from 21.6 to 20.7 , but this was not statistically significant.

\section{$\mathrm{MCH}$ \\ EBEFORE EDURING EAFTER}

25

20

15

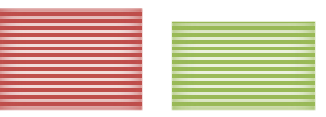

Figure 14.A graphical presentation of $\mathrm{MCH}$ before, during and after ovariohysterectomy.

\section{$\operatorname{MCHC}(\mathrm{g} / \mathrm{dl})$ :}

Mean corpuscular hemoglobin concentration (MCHC) is the ratio of the weight of hemoglobin per cell to the volume in which it is contained. MCHC is calculated by multiplying the hemoglobin concentration by 100 and dividing by the PCV value $M C H C=\frac{H g b \times 100}{P C V}$.

It measures how much hemoglobin is attached to red blood cells (RBCs) generally described as "color of red blood cells". Pale red blood cells are those who have low MCHC. But based on the results of the study, the average results of mean corpuscular hemoglobin concentration (MCHC) are all the same before, during and after ovariohysterectomy and all werewithin the normal range. There were no changes at all.

\section{$\mathrm{MCHC}$}

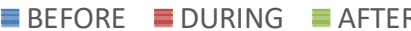

40

30

20

10
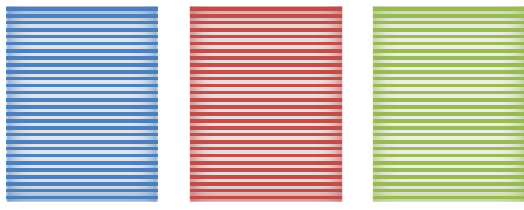

Figure 15.A graphical presentation of MCHC before, during and after ovariohysterectomy.

Platelets $\left(\times 10^{5} / \mu l\right)$ :

The final component that we study when interpreting the $\mathrm{CBC}$, are Platelets. They serve a vital function in the formation of clots in the event of scrape or cut. Based on the results of the study, the platelet count 
appeared normal before ovariohysterectomy (4.8). Considering that during ovariohysterectomy, the animals are having a large cut so a high or elevated (but is still within normal range) platelet count was expected with an average of 6.5 and the count slightly decreased on the following day after ovariohysterectomy (5.6).

\section{PLATELET COUNT}

$$
\text { 를FORE 틈NING EAFTER }
$$

8

6

4

2

0

Figure 16.A graphical presentation of Platelet Count before, during and after ovariohysterectomy.

WBC Count $\left(\times 10^{3} / \mu l\right)$ :

In calculating the white blood cell counts, a general formula was used to convert the count into the number of cells/microliter $\mathrm{WBC}=$

\# of cells counted (average of the two sides)

$\overline{(\text { dilution ratio }) \times(\text { volume of one square }) \times(\text { no.of squares counted })}$

Table 2shows that the WBC count before the surgery was normal (9.8) which means that the animals are fit for surgical operation. During the operation, the WBC counts are too high (16.5); elevated levels of white blood cell count isn't a specific disease, but it can indicate a problem, such as infection, stress, inflammation and trauma,considering that the animals have undergone surgery, elevated level of white blood cell count was anticipated during surgical operation. The good thing was, the white blood cell count returned to normal after these problems are gone, that's why 24 hours after surgery, there was a slight reduction of white blood cell count (14.6) which only means that WBC count was returning to its normal level.

\section{WBC COUNT}

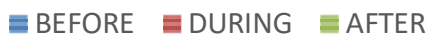

20

15

10

5

0
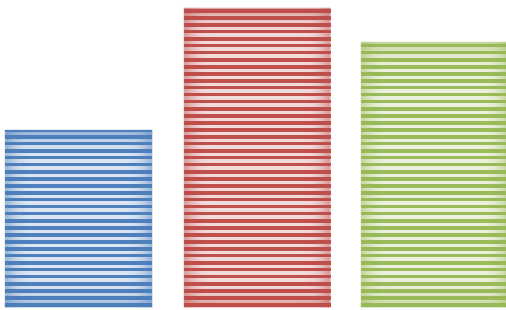

Figure 17.A graphical presentation of WBC Count before, during and after ovariohysterectomy.

\section{DIFFERENTIAL COUNT:}

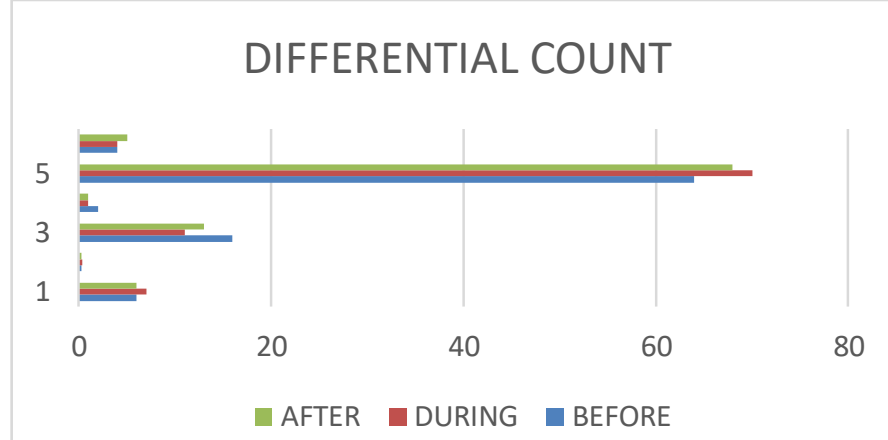

Figure 18. A graphical presentation of the average results of Eosinophils, Basophils, Lymphocytes,

Monocytes and Segmented and Band neutrophils before, during and after ovariohysterectomy.

\section{EOSINOPHILS(\%) and BASOPHILS (\%):}

Eosinophils are large leukocytes with characteristic red granules within the cell structure and comprises 2-10 of the WBCs. It is not uncommon to find no eosinophils in the blood sample of healthy pets, having none is not abnormal.Basophils are similar to eosinophils but are present in much smaller quantities. It is a very uncommon cell in most pets often none are seen.

An increased basophil count typically occurs in conditions that trigger a high eosinophil count. Conversely, eosinophil count often low during periods of stress or inflammation. But based on the results of the study, it could be seen that before, during and after ovariohysterectomy the average eosinophil and basophil counts of the six (6) experimental animals remained within the normal range; no significant changes were observed. 


\section{LYMPHOCYTES(\%):}

Lymphocytes are the second most common white blood cells (WBC) in the body. Based on the results of the study, the lymphocyte counts before ovariohysterectomywas $16 \%$ which is normal. Reduction of lymphocyte counts (11\%) was seen during ovariohysterectomy which falls below the normal range and is termed as Lymphopenia; the most common thing we can see with a stress response. And on post-operative blood examination there was only a very slight increase of lymphocyte counts with an average of $13 \%$ but is still within the normal range.

\section{MONOCYTES(\%):}

Monocytes are often found in higher numbers during stress and also during inflammation. But presented in Table 2 where it could be seen that the monocyte count before surgery was normal with an average of $2 \%$. Whereas, similar counts were seen during and after surgery, $1 \%$. Nonetheless, there are no significant changes were observed since the results of monocyte count before, during and after ovariohysterectomy are all normal.

\section{NEUTROPHILS (segmented\%):}

Neutrophils are the most common white blood cells (WBC). They are the body's first responders. As presented in this figure below, the segmented neutrophils before, during and afterovariohysterectomy are still within the normal range where it was $64 \%$ before surgery, $70 \%$ during surgery and $68 \%$ after surgery.Stress can cause neutrophil level to increase. It could be seen that there was a slight increase of neutrophils during surgery, it may be because of stress, although, the values remained within the normal range.

The average neutrophil numbers are increased during and after ovariohysterectomy, it is usually a sign of stress.

\section{NEUTROPHILS (bands):}

Band cells are immature unsegmented neutrophils. The total band neutrophil counts of the six experimental animals was presented in Table 2 where a similar count were seen before and during ovariohysterectomy and on the post-operative blood examination, a very slight increase of band neutrophils was seen, 5 . The results are within the normal range. No significant changes were observed whichis a good sign, it only means that the segmented neutrophils or the mature neutrophils still outnumbered the band or immature neutrophils.

With the above findings, it means that the animals under study did not suffer from post-operative infection. The slight increase of WBC Count was indicative of surgical stress, a normal reaction of the body from injury.

\section{SUMMARY AND CONCLUSION}

The significance of this study is that, heart rate evaluation and Complete Blood Count (CBC) are examples of the most beneficial opportunities to glean information about the animal's health status during and after surgery. The heart rate and complete blood count results before the six experimental animals are subjected to surgery was invaluable and was presented to make sure that the animals are healthy. Based on the results of the study, the following conclusions were drawn:

The heart rate was still within the reference range throughout the experimental period (before, during and after ovariohysterectomy). The reduction of heart rate during surgery could be attributed to the surgical level of anesthesia.

A tabulated form and a graphical presentation were used to compare the complete blood count (CBC) results of the six experimental animals used in this study before, during and after ovariohysterectomy. No significant changes were observed in the values of the $\mathrm{Hgb}, \mathrm{MCH}, \mathrm{MCHC}$, Eosinophils, Basophils, Monocytes and Band neutrophils but a significant numerical changes were observed in the values of RBC, WBC, PCV, MCV, Platelets, Lympocytes and Segmented neutrophils throughout the experimental period:A significant decrease of red blood cell (RBC) count was noticed from 7.5 (before) to 5.8 (during), although, it was a very slight reduction when compared to the normal range (6-9).Thewhite blood cell (WBC) count increased significantly during ovariohysterectomy from 9.8 (before) to 16.5 (during), although, just a very little increase when compared to the normal range (6-15).A slight decrease in the packed cell volume (PCV) values during ovariohysterectomy was observed, but this was not statistically significant since it was still within the normal range (37-55).Reduction of Mean Corpuscular Volume (MCV) values was observed, however, it does not exceed the normal range. A significant increase in Platelet counts was seen during and after ovariohysterectomy from but the results were still within the normal range. There was a slight reduction in the lymphocyte count during ovariohysterectomy. The increase of segmented neutrophil counts intra and postoperatively were noticed but also, it did not exceed on its normal range.

And that ovariohysterectomy, the same with other surgical procedure also causes intra-operative stress 
that may alter the physiologic profile and trigger operative risk.

\section{RECOMMENDATIONS}

The author would like to recommend that similar study be conducted using other species of experimental animal particularly in cats. It is also recommended that pre-operative evaluation of the surgical patient be given important consideration before ovariohysterectomy in order to avoid intraoperative and post-operative risk. Extra precautions should be given to those surgical patients with abnormal laboratory parameters. It is further recommended to subject the surgical patient for intravenous fluid infusion during the intra-operative and post-operative period to minimize the effect of intra-operative stress.

\section{REFERENCES:}

1. Benjamin, MM (1978). Outline of Veterinary Clinical Pathology. Hematolgy. $3^{\text {rd }}$ Edition. The IOWA State University Press Amees, Iowa U.S.A. pp. 42-112.

2. Bojrab, MJ (1981). Pathophysiology in Small Animal Surgery. Lea and Febiger, Philadelphia, USA. pp. 5-9.

3. Coles, EH (1974). Veterinary Clinical Pathology. $2^{\text {nd }}$ Edition. W.B Saunders Company, Philadelphia, London, Toronto. pp. $1-3$.

4. Cowell, RL, Tylrt, RD and Minkoth, JH (1999). Diagnostic Cytology and Hematology of the Dog and Cat. $2^{\text {nd }}$ Edition. pp.254-257.
5. Ettinger, SJ and Sutter, PF (1970). Canine Cardiology Clinical Examination. W.B Saunders Company. p.3

6. Faulkner, RT (1985). Textbook of Small Animal Surgery. Operative Consideration of Surgical Patient. San Diego, California. Vol.I. pp. 242-249.

7. Giese, Melissa (2017).White Blood Cells in Dogs and Cats. The White Blood Cell Count is Diagnostic Tool for Evaluating Your Pet's Health.

8. Geniusvets (2014). Canine Laboratory Tests for Veterinary Care. Broad Ripple Animal Clinic http://www.bracpet.com/services/canine-laboratory-testsveterinary-care. Accessed: March 2017

9. Kahn, CM (2007). The Merck/Merial Manual for Pet Health Disorders and Diseases of Dogs. Home Edition. Westford, Massachussetes U.S.A. pp. 54-1070.

10. Mandeville, JJ (1985). The Complete Dog Book. Healthy Dog. $17^{\text {th }}$ Edition. pp 647-650.

11. McBride, DE (1995). Veterinary Clinical Laboratory Procedures. The Complete Blood Count. LaGuardia Community College, Long Island City, New York.

12. Rubin, D (2015). Understanding Blood Work: The Complete Blood Count (CBC) for Dogs http://www.petplace.com/article/dogs/diseases-conditions-ofdogs/features/understanding-blood-work-the-completeblood-count-cbc-for-dogs. Accessed: March 2017.

13. Sirois, Margi (1995). Mosby's Fundamentals of Veterinary Technology. Veterinary Clinical Laboratory Procedures. USA.

14. Schalm, OW (1994). Blood Volume and Water Balance. Veterinary Hematology. $3^{\text {rd }}$ Edition.

15. Stockham, SL and Scoot, MA (2002). Fundamentals of Veterinary Clinical Pathology. Basic Hematologic Assay. IOWA State Press. Pp. 31-45.

16. Willard, MD., Tvedten, H and Turnwald, GH (1999). Small Animal Clinical Diagnosis by Laboratory Methods. General Laboratory Concept. Philadelphia London New York St. Louis Sydney Toronto. $3^{\text {rd }}$ edition. pp 1-3. 